\title{
GESUNDHEITS-KRANKHEITSPROZESS IM AMAZONASGEBIET: UMWELTFAKTOREN UND DIE EMERGING VON KRANKHEITEN
}

\section{ARTIKEL ÜBERPRÜFEN}

CUSTÓDIO, Wenderson Picanço ${ }^{1}$, CHAVES, Juliana Vitória Rocha Leite ${ }^{2}$, PANTOJA, Patrícia Trindade ${ }^{3}$, CÁRDENAS, Anneli Mercedes Celis de ${ }^{4}$, ANDRADE, Rosemary Ferreira de ${ }^{5}$, DAPUREZA, Demilto Yamaguchi ${ }^{6}$, FECURY, Amanda Alves ${ }^{7}$

CUSTÓDIO, Wenderson Picanço. Et al. Gesundheits-Krankheitsprozess im Amazonasgebiet: Umweltfaktoren und die Emerging von Krankheiten. Revista Científica Multidisciplinar Núcleo do Conhecimento. Jahr. 06, Hrsg. 12, Vol. 03, S. 05 21. Dezember 2021. ISSN: 2448-0959, Zugangslink: https://www.nucleodoconhecimento.com.br/gesundheit/emerging-von-krankheiten, DOI: 10.32749/nucleodoconhecimento.com.br/gesundheit/emerging-von-krankheiten

\footnotetext{
${ }^{1}$ Master-Abschluss in Gesundheitswissenschaften von der Föderalen Universität Amapá; Facharzt für Hämatologie durch die Brasilianische Union der Hochschulen; Bachelor-Abschluss in Biomedizin von der Fakultät von Macapá; Abschluss in Pädagogik an der City University of São Paulo; Abschluss in Biowissenschaften Universität Stadt São Paulo. ORCID: 0000-0003-0424-6852.

${ }^{2}$ Masterstudent in Gesundheitswissenschaften von der Bundesuniversität Amapá; Postgraduiertenstudium in Kinderzahnheilkunde durch die Gruppe der zahnärztlichen Fachgebiete; Postgraduiertenstudium in Zahnmedizin für Krebspatienten am Syrisch-Libanesischen Krankenhaus; Abschluss in Zahnmedizin von der Fakultät von Macapá - FAMA. ORCID: 0000-0002-7595-9094.

${ }^{3}$ Masterstudent in Gesundheitswissenschaften von der Bundesuniversität Amapá; Postgraduate in Dringlichkeit und Notfall und ICU vom Madre Tereza College-FAMAT; Postgraduiertenstudium in Lehre und

Hochschulmanagement vom Fatec College; Abschluss in Krankenpflege von der Fakultät von Macapá - FAMA. ORCID: 0000-0002-8667-0165.

${ }^{4} \mathrm{PhD}$ in Krankenpflege, School of Nursing, Universität von São Paulo EEUSP, Master in Fundamental Nursing, Universität von São Paulo EEUSP. Abschluss in Krankenpflege an der Nationalen Universität von Callao in Lima Peru. ORCID: https://orcid.org/0000-0002-6581-4326.

${ }^{5}$ Abschluss in Krankenpflege und Geburtshilfe von der Staatlichen Universität Pará (UEPA), Master in Krankenpflege (UFPA) und PhD in Wissenschaft: sozio-ökologische Entwicklung (NAEA / UFPA). Derzeit ist er ordentlicher Professor an der Föderalen Universität Amapá. Er entwickelt Arbeiten zu folgenden Themen: Public Health, Epidemiologie, Amapá, Amazonas, Malaria und Migration. ORCID: https://orcid.org/0000-00034472-8565.

${ }^{6} \mathrm{PhD}$ in Naturwissenschaften, Master in Sport und Abschluss in Sport. ORCID: https://orcid.org/0000-00018336-2178.

${ }^{7} \mathrm{PhD}$ und Master-Abschluss in Tropenkrankheiten, Spezialist für Mikrobiologie, Biomedizinische ORCID: http://orcid.org/0000-0001-5128-8903.
}

RC: 104518

Zugangslink: https://www.nucleodoconhecimento.com.br/gesundheit/emerging-vonkrankheiten 


\section{ZUSAMMENFASSUNG}

Einleitung: Der Amazonas war schon immer Gegenstand zahlreicher Studien, die sich auf seine Umwelt, Gesellschaft und Gesundheit konzentrierten. Forschungsfrage: Welche Umweltfaktoren tragen zur Entstehung von Krankheiten im Amazonasgebiet bei? Ziel: Behandlung der wichtigsten Umweltfaktoren, die zur Entstehung von Krankheiten im Amazonasgebiet beitragen. Methode: Dies ist eine Übersicht über die Literatur in den Datenbanken Scientific Electronic Library Online (SciELO), US National Library of Medicine National Institutes of Health (PubMeD), Virtual Health Library (VHL) und National Institute of Space Research (INPE). Insgesamt wurden bei der Erstellung der Studie 18 Artikel verwendet, die alle die ein- und ausschlusskriterien durchliefen, die festgelegt wurden, um nur Zeitschriften mit thematischen Ansätzen zu filtern. Ergebnisse: Aus epidemiologischer Sicht weist der amazonische Raum, der durch die Interaktion des sozio ökologischen Systems gebildet wird, andere Merkmale auf als andere Teile des Landes. Dieser Unterschied wird durch die natürliche ökologische Grundlage und ihre Formen der Besetzung und Entwicklung verursacht. Schlussfolgerung: Die wichtigsten Umweltfaktoren, die zur Entstehung von Krankheiten im Amazonasgebiet beitragen, sind Entwaldung, Verbrennung, Flussverschmutzung, Urbanisierungsprozess und Agrarindustrie.

Schlüsselwörter: Gesundheits und Krankheits, Amazon, Umweltfaktoren.

\section{EINLEITUNG}

Der Amazonas war schon immer Gegenstand zahlreicher Studien, die sich auf seine Umwelt, Gesellschaft und Entwicklung konzentrierten. In den Veröffentlichungen der letzten Jahrzehnte wurden wichtige regionale Aspekte wie Biodiversität, Landnutzung, Wissenschaft und Technologie,_Stadtwachstum (BROWDER und GODFREY, 2017), nachhaltige Entwicklung und entwaldungs verursachende Entwicklungsprojekte (PERZ, 2002) diskutiert.

Um die Komplexität des Amazonas in Bezug auf die verschiedenen Regionen der Welt zu verstehen, ist es wichtig, sich daran zu erinnern, dass diese Komplexität nicht auf RC: 104518

Zugangslink: https://www.nucleodoconhecimento.com.br/gesundheit/emerging-vonkrankheiten 
seine biologischen und ökologischen Eigenschaften beschränkt ist, sondern mit der Art und Weise zusammenhängt, wie sich die Bevölkerung, die inn bewohnt, in seiner Lebensweise und in der Art des Handelns und Denkens unterscheidet (ROLIM, 2015).

Das Konzept der Gesundheits und Krankheits kann eine regionale Dimension annehmen, da es in dieser Region eine immense Lücke an wissenschaftlichen Informationen über soziale und ökologische Bedingungen gibt, um den Anforderungen der Gesellschaft gerecht zu werden. Es ist erwähnenswert, dass es in dieser Region eine hohe Konzentration von Menschen gibt, die in städtischen Zentren, insbesondere in den Hauptstädten, ansässig sind, was zu weit verbreiteten Problemen in großen Zentren führt, wie zum Beispiel: Mangel an behandeltem Wasser und grundlegenden sanitären Einrichtungen, Verbreitung von Insekten vektoren von Krankheiten, in der Regel anopheline übertragende Malaria, Dengue-Fieber unter anderem (CAÑETE und RAVENA-CAÑETE, 2010).

Die Wahl dieser Region als Gegenstand der Analyse wird durch ihre ökologische (Klima, Ökosysteme und Biodiversität) und demographische (soziale Vielfalt mit geringer Bevölkerungsdichte und Konzentration in Städten) gerechtfertigt, die in Kombination mit wirtschaftlichen Prozessen wie extensiver Landwirtschaft, Viehzucht, Mineralgewinnung, Wasserkraft und Industrie die Gesundheit der Bevölkerung gemäß ihrem Entwicklungsmodell beeinflussen. Darauf aufbauend lautet die Hauptfrage der Studie: Welche Umweltfaktoren tragen zur Entstehung von Krankheiten im Amazonasgebiet bei?

Ziel dieser Studie war es daher, die Umweltfaktoren hervorzuheben, die zur Entstehung von Krankheiten in der Amazonasregion beitragen, sowie die auf die Region ausgerichtete Forschung hervorzuheben, um eine größere bibliographische Sammlung zu diesem Thema zu ermöglichen und die Notwendigkeit der Schaffung und Neuformulierung der öffentlichen Gesundheitspolitik für die in dieser Region lebende Bevölkerung hervorzuheben. 


\section{METHODIK}

Diese Arbeit wurde durch eine Literaturrecherche in wissenschaftlichen Zeitschriften durchgeführt, die auf den Websites und Datenbanken verfügbar ist: Scielo, pubmed, Virtual Health Library und Studien des nationalen Instituts für Weltraumforschung. Die Artikel wurden zwischen August und November 2021 durchsucht, 18 Artikel wurden gesammelt und alle für den Aufbau der Studie ausgewählt. Aufnahmekriterien waren Artikel, die von 2000 bis 2021 in englischer, spanischer und portugiesischer Sprache veröffentlicht wurden. Ausschlusskriterien waren hingegen Vor dem Jahr 2000 veröffentlichte Artikel, Duplikate, Editorials des Herausgeber briefes sowie Zeitschriften in anderen als den in den Einschlusskriterien genannten Sprachen. Die Deskriptoren, die zur Suche in den Studien verwendet wurden, waren: Gesundheits krankheit; Amazon; Umweltfaktoren.

Somit ist dieser Artikel eine Analyse des Gesundheits-Krankheitsprozesses im Amazonasgebiet und der Umweltfaktoren, die die Entstehung von Krankheiten beeinflussen (soziale, wirtschaftliche, kulturelle und Umweltfaktoren und andere Faktoren, die das Auftreten von Gesundheitsproblemen in der Bevölkerung beeinflussen), Nach Sobral und Freitas (2010) unter Verwendung quantifizierbarer Variablen (Indikatoren) und Literaturübersicht.

\section{ERGEBNISSE}

Tabelle 1. Top-Ergebnisse ausgewählter Artikel für die Forschung

\begin{tabular}{|c|c|c|c|}
\hline & Titel & $\begin{array}{l}\text { Autoren, } \\
\text { Erscheinungsjahr } \\
\text { und Land }\end{array}$ & Wichtigste Ergebnisse \\
\hline $\begin{array}{l}\text { ARTIKEL } \\
\text { I }\end{array}$ & $\begin{array}{l}\text { A evolução do } \\
\text { conhecimento } \\
\text { sobre hepatites } \\
\text { virais na região }\end{array}$ & $\begin{array}{l}\text { Bensabath } \quad \text { e } \\
\text { Soares, } 2016, \\
\text { Brasil. }\end{array}$ & 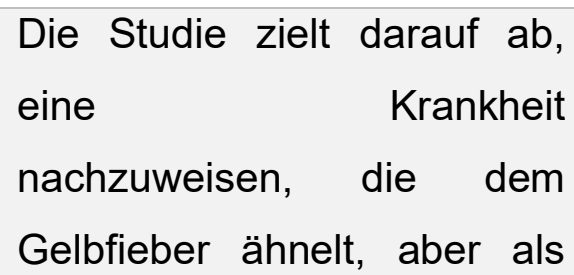 \\
\hline
\end{tabular}

RC: 104518

Zugangslink: https://www.nucleodoconhecimento.com.br/gesundheit/emerging-vonkrankheiten 


\begin{tabular}{|c|c|c|c|}
\hline & $\begin{array}{lr}\text { Amazônica: } & \text { da } \\
\text { epidemiologia } & \text { e } \\
\text { etiologia } & \text { à } \\
\text { prevenção. } & \end{array}$ & & $\begin{array}{l}\text { neue Krankheit betrachtet } \\
\text { wird und in Gebieten der Täler } \\
\text { der Flüsse Juruá, Purus und } \\
\text { Madeira auftritt. Von } \\
\text { Bewohnern wegen hoher } \\
\text { Letalität gefürchtet, klinisch } \\
\text { eine Hepatoenzephalopathie } \\
\text { der fulminanten Evolution } \\
\text { (Mittelwert von } 5 \text { bis } 6 \text { Tagen). }\end{array}$ \\
\hline $\begin{array}{l}\text { ARTIKEL } \\
\text { II }\end{array}$ & $\begin{array}{l}\text { Chagas disease } \\
\text { and globalization } \\
\text { of the Amazon. }\end{array}$ & $\begin{array}{l}\text { Briceño-Léon, } \\
\text { 2007, Brasil. }\end{array}$ & $\begin{array}{l}\text { Der fragliche Artikel wirft } \\
\text { einen Anstieg der Zahl der } \\
\text { autochthonen Fälle der } \\
\text { Chagas-Krankheit den } \\
\text { Amazonasgebiet seit das } \\
\text { 1970er Jahren auf, was zu } \\
\text { Befürchtungen führt, dass die } \\
\text { Krankheit zu einem neuen } \\
\text { Problem der öffentlichen } \\
\text { Gesundheit in der Region } \\
\text { wird.Diese Transformation im } \\
\text { epidemiologischen Muster } \\
\text { der Krankheit } \\
\text { Amazonasgebiet kann durch } \\
\text { die ökologischen und sozialen } \\
\text { Veränderungen der letzten } 30 \\
\text { Jahre erklärt werden. }\end{array}$ \\
\hline $\begin{array}{l}\text { ARTIKEL } \\
\text { III }\end{array}$ & $\begin{array}{l}\text { Rainforest Cities. } \\
\text { Urbanization, } \\
\text { Development and } \\
\text { Colonization of the } \\
\text { Brazilian Amazon. }\end{array}$ & $\begin{array}{l}\text { Browder e Godfrey, } \\
2017, \quad \text { Estados } \\
\text { Unidos. }\end{array}$ & $\begin{array}{l}\text { Die Studie zeigte die } \\
\text { Versöhnung von } \\
\text { Umweltproblemen angesichts } \\
\text { der aktuellen globalen } \\
\text { Urbanisierung angesichts des }\end{array}$ \\
\hline
\end{tabular}

RC: 104518

Zugangslink: https://www.nucleodoconhecimento.com.br/gesundheit/emerging-vonkrankheiten 


\begin{tabular}{|c|c|c|c|}
\hline & & & $\begin{array}{l}\text { intensiven städtischen } \\
\text { Wandels im Amazonasgebiet. }\end{array}$ \\
\hline $\begin{array}{l}\text { ARTIKEL } \\
\text { IV }\end{array}$ & $\begin{array}{l}\text { Populações } \\
\text { Tradicionais } \\
\text { Amazônicas: } \\
\text { revisando } \\
\text { conceitos. }\end{array}$ & $\begin{array}{l}\text { Cañete e Ravena- } \\
\text { Cañete, } \\
\text { Brasil. }\end{array}$ & $\begin{array}{l}\text { Die Arbeit zielte darauf ab, } \\
\text { das Konzept der traditionellen } \\
\text { Bevölkerung im Amazonas- } \\
\text { Kontext kritisch zu diskutieren } \\
\text { und zu überprüfen, ob das } \\
\text { Konzept der traditionellen } \\
\text { Bevölkerung als kollektive } \\
\text { Identität verstanden wird, die } \\
\text { die Besonderheit hat, } \\
\text { Lebensweisen der Integration } \\
\text { und Intimität mit der Umwelt } \\
\text { zu entwickeln. }\end{array}$ \\
\hline $\begin{array}{l}\text { ARTIKEL } \\
\text { V }\end{array}$ & $\begin{array}{l}\text { O trabalho como } \\
\text { determinante do } \\
\text { processo saúde- } \\
\text { doença. }\end{array}$ & $\begin{array}{l}\text { Cardoso, 2015, } \\
\text { Brasil. }\end{array}$ & $\begin{array}{l}\text { Die Studie analysierte, wie im } \\
\text { aktuellen Kontext die } \\
\text { organisatorischen und } \\
\text { physischen Bedingungen der } \\
\text { Arbeit, Situationen und } \\
\text { Arbeitsbeziehungen und } \\
\text { Formen des Managements } \\
\text { die Krankheit der } \\
\text { Arbeitnehmer bestimmen und } \\
\text { dazu beitragen, eine so } \\
\text { komplexe Beziehung zu } \\
\text { verstehen }\end{array}$ \\
\hline $\begin{array}{l}\text { ARTIKEL } \\
\text { VI }\end{array}$ & $\begin{array}{l}\text { Determinante da } \\
\text { saúde no Brasil: a } \\
\text { procura da } \\
\text { equidade na } \\
\text { saúde. }\end{array}$ & $\begin{array}{l}\text { Carrapato et al., } \\
\text { 2017, Brasil. }\end{array}$ & $\begin{array}{l}\text { Die vorliegende Studie zielte } \\
\text { darauf ab, durch Bibliometrie } \\
\text { zu identifizieren, was die } \\
\text { Determinanten der } \\
\text { Gesundheit mit den größten }\end{array}$ \\
\hline
\end{tabular}




\begin{tabular}{|c|c|c|c|}
\hline & & & $\begin{array}{l}\text { Auswirkungen auf die } \\
\text { Gesundheit der Bevölkerung } \\
\text { angesichts der Frage der } \\
\text { Lebensqualität sind, die fast } \\
\text { unmittelbar mit der } \\
\text { Gesundheit verbunden ist. }\end{array}$ \\
\hline $\begin{array}{l}\text { ARTIKEL } \\
\text { VII }\end{array}$ & $\begin{array}{l}\text { The Health of } \\
\text { Indigenous } \\
\text { Peoples in the } \\
\text { Brazilian Amazon. }\end{array}$ & $\begin{array}{l}\text { Confalonieri, 2000, } \\
\text { Amsterdam. }\end{array}$ & 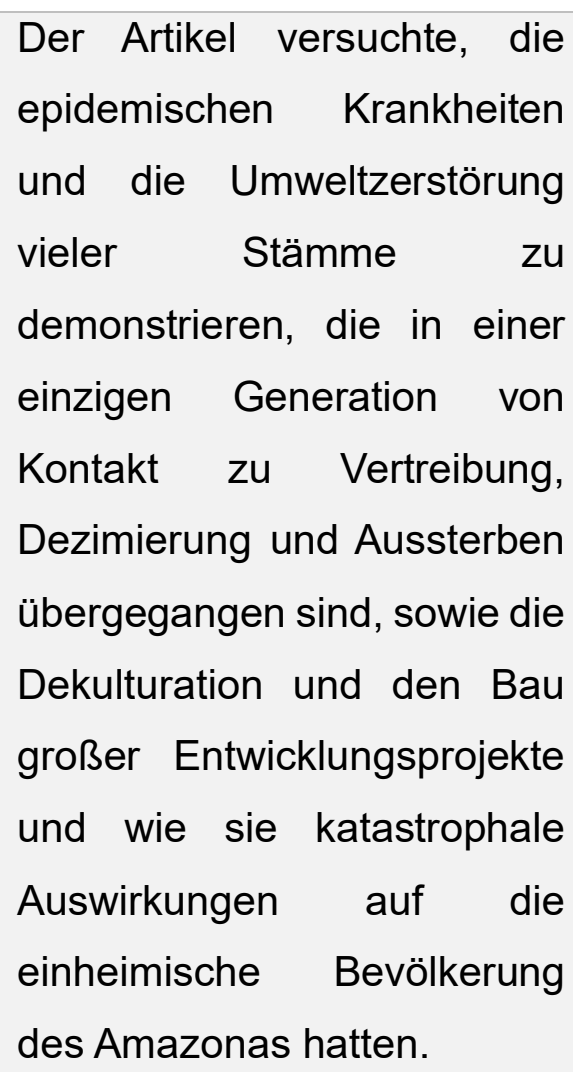 \\
\hline $\begin{array}{l}\text { ARTIKEL } \\
\text { VIII }\end{array}$ & $\begin{array}{l}\text { Saúde na } \\
\text { Amazônia: um } \\
\text { modelo conceitual } \\
\text { para a análise de } \\
\text { paisagens } \\
\text { doenças. }\end{array}$ & $\begin{array}{l}\text { Confalonieri, 2005, } \\
\text { Brasil. }\end{array}$ & $\begin{array}{l}\text { Die Studie überprüfte das } \\
\text { epidemiologische Profil der } \\
\text { amazonasischen } \\
\text { Populationen in Bezug auf die } \\
\text { sozio-ökologische Dynamik } \\
\text { der Region und entwickelte } \\
\text { ein konzeptionelles } \\
\text { Analysemodell, das drei } \\
\text { Hauptkategorien } \\
\text { Landschaften }\end{array}$ \\
\hline
\end{tabular}

RC: 104518

Zugangslink: https://www.nucleodoconhecimento.com.br/gesundheit/emerging-vonkrankheiten 


\begin{tabular}{|c|c|c|c|}
\hline & & & $\begin{array}{l}\text { Amazonasgebiet umfasst: } \\
\text { natürlich, anthropisiert und } \\
\text { konstruiert. }\end{array}$ \\
\hline $\begin{array}{l}\text { ARTIKEL } \\
\text { IX }\end{array}$ & $\begin{array}{l}\text { Indicadores de } \\
\text { sustentabilidade } \\
\text { ambiental e de } \\
\text { saúde na } \\
\text { Amazônia Legal. }\end{array}$ & $\begin{array}{l}\text { Freitas e Giatti, } \\
\text { 2009, Brasil. }\end{array}$ & $\begin{array}{l}\text { Der Artikel zeigte die } \\
\text { aktuellen Herausforderungen } \\
\text { der öffentlichen Gesundheit } \\
\text { auf, die hauptsächlich in der } \\
\text { Strukturierung von } \\
\text { Indikatorsystemen liegt, die } \\
\text { es ermöglichen, die } \\
\text { Bedingungen und Trends der } \\
\text { Umwelt- } \\
\text { Gesundheitsnachhaltigkeit zu } \\
\text { überwachen. Der Artikel } \\
\text { konzentrierte sich darauf, zu } \\
\text { zeigen, wie man dieser } \\
\text { Herausforderung begegnen } \\
\text { kann, wobei der Schwerpunkt } \\
\text { der Analyse auf den } \\
\text { Mitgliedstaaten der LegalEn } \\
\text { Amazonas-Makroregion lag. }\end{array}$ \\
\hline $\begin{array}{l}\text { ARTIKEL } \\
\mathbf{X}\end{array}$ & $\begin{array}{l}\text { Custo das } \\
\text { doenças } \\
\text { atribuíveis a } \\
\text { fatores ambientais } \\
\text { na cidade de } \\
\text { Manaus, } \\
\text { Amazonas, Brasil. }\end{array}$ & $\begin{array}{l}\text { Medeiros, } 2014, \\
\text { Brasil. }\end{array}$ & $\begin{array}{l}\text { Die Studie präsentierte die } \\
\text { Schätzung der Kosten von } \\
\text { Krankheiten, die auf } \\
\text { Umweltfaktoren in der Stadt } \\
\text { Manaus zwischen } 1998 \text { und } \\
2009 \text { zurückzuführen sind, } \\
\text { zeigte die Ursachen von } \\
\text { Krankenhausaufenthalten } \\
\text { und wie sie auf der Grundlage } \\
\text { der Studien der globalen }\end{array}$ \\
\hline
\end{tabular}

RC: 104518

Zugangslink: https://www.nucleodoconhecimento.com.br/gesundheit/emerging-vonkrankheiten 


\begin{tabular}{|c|c|c|c|}
\hline & & & $\begin{array}{l}\text { Krankheitslast und der } \\
\text { vergleichenden } \\
\text { Risikoanalyse } \\
\text { Weltgesundheitsorganisation } \\
\text { gruppiert wurden. }\end{array}$ \\
\hline $\begin{array}{l}\text { ARTIKEL } \\
\text { XI }\end{array}$ & $\begin{array}{l}\text { Recent Progress } \\
\text { in Modeling } \\
\text { Biome-Climate } \\
\text { Interactions in } \\
\text { Amazônia. }\end{array}$ & $\begin{array}{l}\text { Nobre, } \\
\text { Brasil. }\end{array}$ & $\begin{array}{l}\text { Die Forschung zielte darauf } \\
\text { ab, die Beziehung zwischen } \\
\text { historischer Entwaldung und } \\
\text { Niederschlag auf } \\
\text { verschiedenen geografischen } \\
\text { Skalen im südbrasilinischen } \\
\text { Amazonasgebiet (SBA) zu } \\
\text { untersuchen.Die } \\
\text { Auswirkungen von Szenarien } \\
\text { der Entwaldungspolitik auf die } \\
\text { Landwirtschaft in der Region } \\
\text { wurden ebenfalls bewertet. }\end{array}$ \\
\hline $\begin{array}{l}\text { ARTIKEL } \\
\text { XII }\end{array}$ & $\begin{array}{l}\text { Population Growth } \\
\text { and Net Migration } \\
\text { in the Brazilian } \\
\text { Legal Amazon, } \\
\text { 1970- 1996, em } \\
\text { WOOD e PORRO. }\end{array}$ & $\begin{array}{l}\text { Perz, } 2002, \\
\text { Estados Unidos. }\end{array}$ & $\begin{array}{l}\text { Der Artikel basierte auf Daten } \\
\text { aus der brasilianischen } \\
\text { demografischen } \\
\text { Volkszählung, um zu zeigen, } \\
\text { wie die Bevölkerung im } \\
\text { Amazonasgebiet zwischen } \\
1970 \text { und } 1996 \text { wuchs, was } \\
\text { zur Entwaldung führte, wobei } \\
\text { der Schwerpunkt darauf lag, } \\
\text { wie die Migration zu diesem } \\
\text { Wachstum beitrug. Es wurde } \\
\text { analysiert, wo sich das } \\
\text { Bevölkerungswachstum für } \\
\text { die Region im Allgemeinen }\end{array}$ \\
\hline
\end{tabular}

RC: 104518

Zugangslink: https://www.nucleodoconhecimento.com.br/gesundheit/emerging-vonkrankheiten 


\begin{tabular}{|c|c|c|c|}
\hline & & & $\begin{array}{l}\text { sowie für ihre ländlichen und } \\
\text { städtischen Komponenten } \\
\text { konzentriert. }\end{array}$ \\
\hline $\begin{array}{l}\text { ARTIKEL } \\
\text { XIII }\end{array}$ & $\begin{array}{lr}\text { Estudo dos } \\
\text { arbovirus } \\
\text { Amazônia } \\
\text { Brasileira. }\end{array}$ & $\begin{array}{l}\text { Rodrigues, 2004, } \\
\text { Brasil. }\end{array}$ & $\begin{array}{l}\text { Die Forschung versuchte, die } \\
\text { von St. Louis (SLE), Ost } \\
\text { (EWR), West (WEE) und } \\
\text { venezolanische } \\
\text { Pferdeenzephalitis, [subtipos } \\
\text { III, Mucambo (MUC) e IV, } \\
\text { Pixuna (PIX)] resultierend aus } \\
\text { Studien, die in verschiedenen } \\
\text { Gebieten des brasilianischen } \\
\text { Amazonasgebiets } \\
\text { durchgeführt } \\
\text { insbesondere entlang von } \\
\text { Autobahnen } \\
\text { Entwicklungsprojekten. }\end{array}$ \\
\hline $\begin{array}{l}\text { ARTIKEL } \\
\text { XIV }\end{array}$ & $\begin{array}{l}\text { A pobreza e a } \\
\text { riqueza na região } \\
\text { amazônica e a } \\
\text { contribuição da } \\
\text { política } \\
\text { assistência social: } \\
\begin{array}{lr}\text { estado do } \\
\text { amazonas } \\
\text { foco. }\end{array}\end{array}$ & $\begin{array}{l}\text { Rolim, } \\
\text { Brasil. }\end{array}$ & $\begin{array}{l}\text { Der Artikel befasste sich mit } \\
\text { den Widersprüchen zwischen } \\
\text { Armut und Reichtum und dem } \\
\text { Beitrag der Sozialhilfepolitik } \\
\text { im Bundesstaat Amazonas. } \\
\text { Es zeigte sich als Ergebnis, } \\
\text { dass das gegenwärtige } \\
\text { Manaus, die Hauptstadt des } \\
\text { Staates, mit den } \\
\text { Widersprüchen der neuen } \\
\text { kapitalistischen Ordnung } \\
\text { koexistiert, in der das } \\
\text { Nebeneinander von Reichtum } \\
\text { und Armut visualisiert wird. }\end{array}$ \\
\hline
\end{tabular}

RC: 104518

Zugangslink: https://www.nucleodoconhecimento.com.br/gesundheit/emerging-vonkrankheiten 


\begin{tabular}{|c|c|c|c|}
\hline $\begin{array}{l}\text { ARTIKEL } \\
\text { XV }\end{array}$ & $\begin{array}{l}\text { Modelo de } \\
\text { organização de } \\
\text { indicadores para } \\
\text { operacionalização } \\
\text { dos determinantes } \\
\text { socioambientais } \\
\text { da saúde. }\end{array}$ & $\begin{array}{l}\text { Sobral e Freitas, } \\
\text { 2010, Brasil. }\end{array}$ & $\begin{array}{l}\text { Die Studie verifizierte die } \\
\text { sozialen Determinanten und } \\
\text { ihre Beziehungen zum } \\
\text { Gesundheits- } \\
\text { Krankheitsprozess zwischen } \\
\text { verschiedenen } \\
\text { Bevölkerungsgruppen und } \\
\text { ihre große Relevanz für die } \\
\text { öffentliche Gesundheit und } \\
\text { wie sie immer mehr an } \\
\text { Bedeutung gewinnen. In } \\
\text { diesem Prozess wurden } \\
\text { theoretisch-konzeptionelle } \\
\text { Modelle verwendet, um zu } \\
\text { erklären, wie soziale } \\
\text { Determinanten } \\
\text { gesundheitliche } \\
\text { Ungleichheiten erzeugen. }\end{array}$ \\
\hline $\begin{array}{l}\text { ARTIKEL } \\
\text { XVI }\end{array}$ & $\begin{array}{l}\text { Saúde ambientale } \\
\text { desenvolvimento } \\
\text { na Amazônia } \\
\text { legal: indicadores } \\
\text { socioeconômicos, } \\
\text { ambientais } \\
\text { sanitários, } \\
\text { desafios } \\
\text { perspectivas. }\end{array}$ & $\begin{array}{l}\text { Viana et al., 2016, } \\
\text { Brasil. }\end{array}$ & $\begin{array}{l}\text { Der Artikel versuchte, die } \\
\text { Prozesse der sozio- } \\
\text { ökologischen Bestimmung in } \\
\text { den Staaten, die den legalen } \\
\text { Amazonas bilden, auf der } \\
\text { Grundlage der Analyse von } \\
\text { sozioökonomischen, Umwelt- } \\
\text { und Gesundheitsindikatoren } \\
\text { zu diskutieren. }\end{array}$ \\
\hline $\begin{array}{l}\text { ARTIKEL } \\
\text { XVII }\end{array}$ & $\begin{array}{l}\text { Saúde, ambiente } \\
\text { e } \\
\text { desenvolvimento } \\
\text { econômico na }\end{array}$ & $\begin{array}{l}\text { Vincentin } \\
\text { Minayo, } \\
\text { Brasil. }\end{array}$ & $\begin{array}{l}\text { Die Studie analysierte den } \\
\text { Prozess der Transformation } \\
\text { des epidemiologischen } \\
\text { Musters einer amazonischen }\end{array}$ \\
\hline
\end{tabular}

RC: 104518

Zugangslink: https://www.nucleodoconhecimento.com.br/gesundheit/emerging-vonkrankheiten 


\begin{tabular}{|c|c|c|c|}
\hline & Amazônia. & & $\begin{array}{l}\text { Bevölkerung, die an der } \\
\text { Entwicklung von Mineração } \\
\text { Rio do Norte S beteiligt ist.A. } \\
\text { (MRN), in der Gemeinde } \\
\text { Oriximiná (PA), in der } \\
\text { Ortschaft Porto Trombetas. } \\
\text { Die Bestimmung des } \\
\text { Gesundheits- } \\
\text { Krankheitsprozesses wurde } \\
\text { entsprechend } \\
\text { besonderen Bedingungen der } \\
\text { sozialen Reproduktion des } \\
\text { Unternehmens entwickelt, die } \\
\text { sich deutlich von denen der } \\
\text { Bevölkerungen } \\
\text { unterscheiden, die mit der } \\
\text { traditionellen } \\
\text { sozioökonomischen } \\
\text { Entwicklung der Region } \\
\text { verbunden sind. }\end{array}$ \\
\hline $\begin{array}{l}\text { ARTIKEL } \\
\text { XVIII }\end{array}$ & $\begin{array}{l}\text { Deforestation, } \\
\text { Hunting and the } \\
\text { Ecology of } \\
\text { Microbial } \\
\text { Emergence }\end{array}$ & $\begin{array}{l}\text { Wolfe, } 2000, \\
\text { Estados Unidos. }\end{array}$ & $\begin{array}{l}\text { Die Studie versuchte zu } \\
\text { verstehen, wie neue Mikroben } \\
\text { in die menschliche Population } \\
\text { gelangen, wie oft Mikroben je } \\
\text { nach der Bestimmung durch } \\
\text { die Vielfalt der in der Umwelt } \\
\text { vorhandenen Mikroben } \\
\text { entstehen, sowie den Grad } \\
\text { des Kontakts zwischen einer } \\
\text { potenziellen mikrobiellen } \\
\text { Vielfalt des Wirts und der }\end{array}$ \\
\hline
\end{tabular}

RC: 104518

Zugangslink: https://www.nucleodoconhecimento.com.br/gesundheit/emerging-vonkrankheiten 
Anfälligkeit des neuen Wirts für Infektionen.

Quelle: eigene Autoren.

Unter den ausgewählten Artikeln zeigte die überwiegende Mehrheit der Studien, dass die ökologischen und soziologischen Faktoren, die seit 1970 zur Entstehung von Krankheiten im Amazonasgebiet beitragen, wie im Artikel "Chagas-Krankheit und Globalisierung des Amazonas" des Autors Briceño-Léon (2007) gezeigt, dieses historische Einzugsgebiet ausmachen. Dieser Wandel in der Epidemiologie der Krankheiten im Amazonasgebiet lässt sich durch ökologische und soziale Veränderungen in den letzten 30 Jahren erklären, wie der Autor erläutert.

Die Autoren Browder und Godfrey (2017), Browder und Godfrey (2017), Cardoso (2015), Carrapato et al. (2017), Confalonieri (2000), Confalonieri (2005), Medeiros (2014), Nobre (2004), Perz (2002), Sobral und Freitas (2010) und Viana et al. (2016) zeigte in seiner veröffentlichten Forschung zusammenfassend die Beziehung von sozialen und ökologischen Determinanten und ihre intrinsische Verbindung mit dem Gesundheits-Krankheitsprozess verschiedener Bevölkerungsgruppen, ihre signifikante Relevanz für die öffentliche Gesundheit und wie sie stärker in den Vordergrund traten. In diesem Prozess der jahrelangen Geschichten, die der Amazonas mit sozialen Veränderungen und physischen und territorialen Raum konfrontiert hat, und als Folgen die Verschlimmerung von Krankheiten konzentriert sich in dieser Region.

\section{LITERATURRECHERCHE UND DISKUSSION}

\subsection{REGIONALE ASPEKTE}

Ein sehr wichtiges Phänomen in der Amazonas-Umgebung ist die Wechselwirkung zwischen der Atmosphäre und den Wäldern, die eine wichtige Rolle bei der Erzeugung von Niederschlägen in der Region spielen. Das Klima ist im Allgemeinen heiß und feucht und die Temperatur ändert sich nicht viel, da es ein wichtiger Regulator RC: 104518

Zugangslink: https://www.nucleodoconhecimento.com.br/gesundheit/emerging-vonkrankheiten 
biologischer Prozesse ist, insbesondere derjenigen, die mit Infektionskrankheiten zusammenhängen (NOBRE, 2004).

Ein weiterer damit zusammenhängender biophysikalischer Aspekt betrifft die Biologische Vielfalt, insbesondere die Tiervielfalt. Einige Prozesse der fokalen Infektion in diesem Bereich, insbesondere die Prozesse der Virusinfektion, hängen von blutsaugenden Insekten (Vektoren) für die Übertragung (Arbovirus) ab. Je größer die Vielfalt der Tierarten (Wirbeltiere und Wirbellose) in einem bestimmten Gebiet ist, desto größer ist das Risiko eines neuen Wolfe-Infektionsprozesses (2000). Um diesen Punkt zu veranschaulichen, wurden im Amazonasgebiet etwa 196 Arboviren identifiziert, von denen 32 Menschen infizieren können (RODRIGUES, 2004).

Nobre (2004) weist darauf hin, dass in Bezug auf die amazonasische Gesellschaft die folgenden wichtigen demografischen Aspekte hervorgehoben werden können:

Ich. Geringe regionale Bevölkerungsdichte (ca. 20 Millionen Einwohner auf einer Fläche von 3,5 Millionen Quadratkilometern) (INPE, 2013; IBGE, 2013);

II. die hohe Wachstumsrate der Zuwanderung in den letzten 30 Jahren. Es gibt auch eine starke interregionale Migration (PERZ, 2002);

III. Städte sind stark konzentriert, vor allem in Hauptstädten. Ungefähr $70 \%$ der Amazonas-Bevölkerung lebt in großen und kleinen Städten. Charakteristisch ist die rasche, spontane und ungeordnete Entstehung vieler dieser kleinen städtischen Siedlungen ohne die notwendige sanitäre Infrastruktur (BROWDER und GODFREY, 2017);

IV. Es gibt eine große Anzahl traditioneller Populationen (Indianer, Uferbewohner, Gummizapfer usw.). die von Extraktivismus und kleinbäuerlicher Landwirtschaft leben (INPE, 2013; IBGE, 2013).

Ein weiteres wichtiges regionales Merkmal betrifft die Landnutzungs praktiken und ihre ökologischen und sozialen Auswirkungen. Einige dieser Praktiken führen zu Umweltveränderungen, die Gesundheitsrisiken darstellen. Dies ist der Fall bei der RC: 104518

Zugangslink: https://www.nucleodoconhecimento.com.br/gesundheit/emerging-vonkrankheiten 
Quecksilberverschmutzung in Bergwerken; aufgrund des Mangels an sanitärer Infrastruktur in städtischen Gebieten ist wasser durch Mikroorganismen kontaminiert; Rauch vom Brennen nach der Entwaldung verursacht Atemwegsprobleme; lokale Veränderungen im Wasserkreislauf, die Mücken brutstätten hervorbringen, um nur einige weitere direkte Auswirkungen aufzuzählen (CONFALONIERI, 2000).

Es sollte auch daran erinnert werden, dass soziale Konflikte, zum Beispiel Landbesitz, zu Gewalt und kulturellen Konflikten führen, und die drastischen Auswirkungen isolierter indigener Völker und die Einführung mikrobieller Erreger, die diesen Gemeinschaften bisher unbekannt waren, was zur Entstehung neuer Krankheiten führt und die Zahl der Fälle bestehender Krankheiten in der Region erhöht (RODRIGUES, 2004).

Der gemeinsame Hintergrund dieser Ereignisse ist, dass Ressourcen Entwicklungsund Nutzungs Politiken das regionale Potenzial ignorieren, die ökologische Widerstandsfähigkeit und ökologische Nachhaltigkeit des Amazonas vergessen, nicht zu traditioneller Kultur oder Einkommensverteilung führen und sich nicht um Gesundheitsrisiken kümmern (CONFALONIERI, 2000).

In der Praxis geschieht dies sowohl auf Initiative der Regierung als auch durch die Umsetzung großer Infrastrukturprojekte (Autobahnen, Wasserkraftwerke usw.). und Industrie (kommerzieller Bergbau), Förderung der Viehzucht (und Entwaldung), sowie durch spontane extraktive Aktivitäten wie Bergbau, Fischerei, Holzeinschlag und andere (BRICEÑO-LEÓN, 2007; VICENTIN und MINAYO, 2003; FREITAS und GIATTI, 2009).

\subsection{SOZIAL-ÖKOLOGISCHE INDIKATOREN}

In dem Raum, der die südlichen und östlichen Teile des Amazonas (Bundesstaaten Rondônia, Mato Grosso, Tocantins, Maranhão und Pará) umgibt, haben sich in den letzten Jahren Brändausbrüche, akkumulierte Entwaldung und der Bau von Autobahnen sowie Anbaugebiete und Intensivierung des Pestizidverbrauchs intensiviert (INPE, 2013; IBGE, 2013).

RC: 104518

Zugangslink: https://www.nucleodoconhecimento.com.br/gesundheit/emerging-vonkrankheiten 
Diese Ressourcen wurden von Nobre (2004) "Arc of Fire" genannt. In Bezug auf den Verbrauch von Pestiziden waren die mit diesen Substanzen in der Region erzielten Raten ausdrucksstark und machten 18\% der nationalen Gesamtmenge aus. Nach Angaben des brasilianischen Instituts für Geographie und Statistik (IBGE) aus dem Jahr 2009 erreichte der Bundesstaat Mato Grosso die höchste nationale Rate des Pestizideinsatzes pro Hektar Anbaufläche im legalen Amazonasgebiet (IBGE, 2013).

Nach den untersuchten Indikatoren erreichten die Daten über die Zunahme von Ackerland und Weiden auf Naturgebieten und die kumulative Entwaldung im Amazonasgebiet die höchsten Raten im selben "Feuerbogen", mit einer klaren Konvergenz zwischen der Ausweitung der extensiven Landwirtschaft und der Entwaldung zusammen mit dem Auftreten von Bränden und dem Einsatz von Pestiziden (CARDOSO, 2015).

Nach der Argumentation und Rettung von Confalonieri (2005) ist es möglich zu zeigen, wie sehr amazonische Gesellschaften Schwierigkeiten beim Zugang zu Gesundheit und folglich mangelnder Versorgung und Krankheiten haben. Der Autor lenkt die Aufmerksamkeit auf Faktoren wie: geringe regionale demografische Dichte, signifikantes Wachstum der Migration, einschließlich interregionaler Migrationen, hohe Konzentration von Städten, insbesondere in Hauptstädten, mit einem Mangel an sanitärer Infrastruktur und einer großen Anzahl traditioneller Bevölkerungen, schadet den sozialen Determinanten im Amazonasgebiet und verursacht ernsthafte Gesundheitsprobleme.

Für die Autoren Medeiros et al. (2014) wurde auch die Situation der Arbeitsbedingungen in den Bereichen der natürlichen und anthropischen Landschaft des Amazonas diskutiert. Dies liegt daran, dass die Bedingungen und das Arbeitsumfeld Determinanten für arbeitsbedingte Gesundheits-Krankheitsprozess ind und als nicht übertragbare Krankheiten eingestuft werden. Daher wird die Gesundheit des Arbeiters wie folgt konzipiert:

Um campo de saúde pública que tem como objeto de estudo e intervenção as relações produção-consumo e o processo saúde-doença. Neste campo, o trabalho é RC: 104518

Zugangslink: https://www.nucleodoconhecimento.com.br/gesundheit/emerging-vonkrankheiten 
considerado como eixo organizador da vida social, espaço de dominação e resistência dos trabalhadores e determinantes das condições de vida e saúde das pessoas (BRASIL, 2018, p. 136).

Arbeit ist eine wichtige Determinante für das Leben und die Gesundheit der Menschen. In dem Wissen, dass es in der Amazonasregion viele verschiedene Aktivitäten, Beziehungen und Servicebedingungen gibt, ist es wichtig, dass Angehörige der Gesundheitsberufe die Krankheit im Zusammenhang mit den Aufgaben, die in der Amazonasregion ausgeführt werden, fördern, verhindern und diagnostizieren (CARDOSO, 2015).

In diesen sehr spezifischen beruflichen Aktivitäten des Amazonas, die in anderen Teilen Brasiliens selten sind, kann erwähnt werden: Pflanzenextraktivismus - Beruf, der unter anderem extraktive açaí, Gummiverdichter, Paranusssammler, Piloten von Passagiertransportbooten, Goldsucher, Fischer und viele mehr umfasst. Es wird auch wahrgenommen, dass stark erniedrigende Arbeitsbeziehungen, die vielerorts als analog zur Sklaverei angesehen werden können, in der Region aufgrund der Schwierigkeit, die große Landausdehnung im Amazonasgebiet zu kontrollieren, ziemlich offensichtlich sind.

Da es sich um ein erzreiches Gebiet handelt, siedeln sich Prospektoren in dieser Region und in Schutzgebieten an, in denen der Bergbau verboten ist. Infolge des Eindringens in Waldgebiete sind sowohl Prospektoren als auch Extraktoren von Paranüssen und Kautschukzapfern von bakteriellen Krankheiten betroffen, die durch Vektoren oder nicht übertragbare und protozoen, die im Wald existieren, übertragen werden.

Unter innen kann man die Chagas-Krankheit, Malaria, Leishmaniose und viele Viruserkrankungen in der Region erwähnen. Angesichts der großen Zahl dieser Fachkräfte und der Risiken, denen sie ausgesetzt sind, sollte das Gesundheitssystem daher Maßnahmen ergreifen, damit Krankheiten, die direkt oder indirekt mit der Arbeit zusammenhängen, nicht zu einem Faktor erhöhter Morbidität und Mortalität im Amazonasgebiet werden (CARDOSO, 2015).

RC: 104518

Zugangslink: https://www.nucleodoconhecimento.com.br/gesundheit/emerging-vonkrankheiten 
Es ist wichtig hervorzuheben, dass die Debatte über die Arbeit des Amazonas und seinen Umfang sehr reichhaltig ist, da sie auch zeigt, dass der Amazonas ein Ort ist, der noch ein neuer Ort spezifischer Forschung und Gesundheitspolitik zu Lebensbedingungen, Gesundheitskrankheiten und Arbeit in der Region ist (BENSABATH und SOARES, 2016).

\subsection{REGIONALE EPIDEMIOLOGISCHE INDIKATOREN}

In Bezug auf regionale epidemiologische Indikatoren gibt es einige wichtige Unterschiede zwischen der Amazonasregion und dem Rest des Landes. 1996 war die Rate der Krankenhausaufenthalte wegen infektiöser und parasitärer Erkrankungen sehr hoch (12,7\%), viel höher als der nationale Durchschnitt $(8,3 \%)$. Obwohl die Inzidenz dieser Läsionen im Amazonasgebiet abgenommen hat, war die Sterblichkeitsrate durch diese Krankheiten nicht die gleiche (SUDAM, 2000).

Diese Region beherbergt die überwiegende Mehrheit der Malariafälle im Land (98\%), etwa 35\% der Leprafälle im Jahr 2016 und die zweithöchste Inzidenz von Tuberkulose in allen Regionen des Landes. Es ist auch ein sehr endemisches Gebiet der Virushepatitis mit häufigen Ausbrüchen, insbesondere im westlichen Amazonasgebiet (BENSABATH und SOARES, 2016).

Es ist bemerkenswert, dass aufgrund der geografischen Bedingungen der Region sowie des Prozesses der Urbanisierung und des Mangels an sanitären Grundversorgung zum Prozess der Krankheiten beigetragen haben, die im Amazonasgebiet wieder auftauchten, wie Cholera im Jahr 1991 und Dengue Mitte der 1990er Jahre, zusätzlich zu diesen, verursachen andere Krankheiten auch eine große Anzahl von Todesfällen in der Bevölkerung der Region wie Malaria. Leishmaniose tegumentäre amerikanische parasitäre Infektionen und insbesondere Tuberkulose (VIANA et al., 2016).

Die Literatur zeigt auch den unverhältnismäßigen Zusammenhang zwischen verbesserter Lebensqualität und Umweltzerstörung, d.h. Entwicklungsfortschritten in nicht nachhaltiger Weise, und unterstreicht die Bedeutung der Berücksichtigung der RC: 104518

Zugangslink: https://www.nucleodoconhecimento.com.br/gesundheit/emerging-vonkrankheiten 
Kosten von durch Umweltfaktoren verursachten Krankheiten, die im Amazonasgebiet zugenommen haben (MEDEIROS et al., 2014).

Häkchen; Correa und Garcia (2017) brachten Lebensqualität sofort mit Gesundheit in Verbindung. Die Autoren betonen, dass es neben biomedizinischen Modellen einige entscheidende Faktoren gibt, die die persönliche Gesundheit entscheidend beeinflusst haben oder beeinflussen werden. Daher haben diese Determinanten derzeit einen größeren Einfluss auf die Gesundheit als diejenigen, die derzeit von der Medizin abgedeckt werden. Sie betonten die Existenz von drei Determinanten: Umwelt, Wirtschaft und Gesellschaft, für sie sind soziale Determinanten weitgehend für die Ungerechtigkeit des Zugangs zur Gesundheitsversorgung verantwortlich.

\section{SCHLUSSFOLGERUNG}

Basierend auf der Leitfrage der Studie ist es möglich zu folgern, dass die wichtigsten Umweltfaktoren, die zur Entstehung von Krankheiten im Amazonasgebiet beitragen, Entwaldung, Verbrennung, Flussverschmutzung, Urbanisierungsprozess und Agrarindustrie sind, so dass die Diskussion über die Studie schließlich einige Probleme identifiziert hat, die für die Anzahl der Krankheiten im Amazonasgebiet verantwortlich sind. wo man zum Beispiel die geografischen, ethnischen, kulturellen und politischen Aspekte der Gesundheit im Amazonasgebiet berücksichtigen sollte. Es wird darauf hingewiesen, dass es an öffentlichen Maßnahmen mangelt, die der Nachfrage nach Gesundheits und Krankheits im Zusammenhang mit den sozio-ökologischen Indikatoren der Region entsprechen, sowie die Notwendigkeit, weitere Studien und Forschungen durchzuführen, um die Widrigkeiten zu kennen, mit denen diese Bevölkerungsgruppen konfrontiert sind.

Es wird auch darauf hingewiesen, dass die wichtigsten Umweltfaktoren, die zur Entstehung von Krankheiten im Amazonasgebiet beitragen, Entwaldung, Verbrennung, Flussverschmutzung, Urbanisierungsprozess und Agrarindustrie sind, dh alles aus der Aktion des Menschen 
Die Studie zeigt jedoch auch, dass aus der Perspektive der Umweltindikatoren für Gesundheit und Krankheit der Amazonasraum, der durch die Interaktion des sozio ökologischen Systems gebildet wird, unterschiedliche Merkmale von anderen Teilen des Landes aufweist. Dieser Unterschied wird durch die natürliche ökologische Grundlage und ihre Formen der Besetzung und Entwicklung verursacht.

\section{VERWEISE}

BENSABATH, G. und SOARES, M. C. P. Die Entwicklung des Wissens über Virushepatitis im Amazonasgebiet: von der Epidemiologie und Ätiologie bis zur Prävention. Rev. Soc. Bras. Med. Trop., 37(supl. II):14-36, 2016.

BRASIL. Ministério da Saúde. Secretaria de Atenção à Saúde. Secretaria de Vigilância em Saúde. Saúde do trabalhador e da trabalhadora [recurso eletrônico]. Cadernos de Atenção Básica, n. 41 - Brasília: Ministério da Saúde, 2018. 136 p.: il.

BRICEÑO-LEÓN, R. Chagas disease and globalization of the Amazon. Cadernos de Saúde Pública, Rio de Janeiro, v. 23, n. 53, p. 33-40, 2007. Suplemento 1.

BROWDER, J. O. e GODFREY, B. J. Rainforest Cities. Urbanization, Development and Colonization of the Brazilian Amazon. Columbia University Press, New York, 2017, $429 \mathrm{pp}$.

CAÑETE, T. M. R..; RAVENA-CAÑETE, V. Populações Tradicionais Amazônicas: revisando conceitos. In: V Encontro Nacional da Associação Nacional de PósGraduação e Pesquisa em Ambiente e Sociedade, 2010, Florianópolis Anais, V Encontro Nacional da Associação Nacional de Pós-Graduação e Pesquisa em Ambiente e Sociedade, 2010.

CARDOSO, A. C. M. O trabalho como determinante do processo saúde-doença. Tempo soc., São Paulo, v. 27, n. 1, p. 73-93, junho de 2015. 
CARRAPATO, P.; CORREIA, P.; GARCIA, B. Determinante da saúde no Brasil: a procura da equidade na saúde. Saúde soc., São Paulo, v. 26, n. 3, p. 676-689, Sept. 2017.

CONFALONIERI, U. E. C. (org.). The Health of Indigenous Peoples in the Brazilian Amazon. Background paper for the World Bank. Royal Tropical Institute, Amsterdam, 2000, $218 \mathrm{pp}$.

CONFALONIERI, U. E. C. Saúde na Amazônia: um modelo conceitual para a análise de paisagens e doenças. Estud. av., São Paulo, v. 19, n. 53, p. 221-236, Apr. 2005.

FREITAS, C. M.; GIATTI, L. L. Indicadores de sustentabilidade ambiental e de saúde na Amazônia Legal. Cadernos de Saúde Pública, Rio de Janeiro, v. 25, n. 6, p. 1251-1266, 2009.

IBGE - INSTITUTO BRASILEIRO DE GEOGRAFIA E ESTATÍSTICA. Contas nacionais: 2011. Rio de Janeiro, RJ, 2013.

INPE - Instituto Nacional De Pesquisas Espaciais. Monitoramento da floresta amazônica brasileira por satélite: 1988/2011. 2013.

MEDEIROS, M. S. de et al. Custo das doenças atribuíveis a fatores ambientais na cidade de Manaus, Amazonas, Brasil. Ciênc. Saúde coletiva [online]. 2014, vol.19, n.2, pp.599-608. 2014.

NOBRE, C. A. Recent Progress in Modeling Biome-Climate Interactions in Amazônia. Resumo 19.2. Anais III Conf. Cient.do LBA, 27-29 de julho, 2004. Brasília, DF, Cd-Rom.

PERZ, S. Population Growth and Net Migration in the Brazilian Legal Amazon, 1970- 1996, em WOOD e PORRO. Deforestation and Land Use in the Amazon. Gainesville, University Press of Florida, 2002, pp. 95-106. 
RODRIGUES, S. G. Estudo dos arbovirus na Amazônia Brasileira. Em: Resumos do III Simpósio Internacional sobre Arbovirus nos Trópicos e Febres Hemorrágicas, Belém, Pará, 30/11 - 3/12, 2004. Instituto Evandro Chagas, 2004.

ROLIM, D. C. A pobreza e a riqueza na região amazônica e a contribuição da política de assistência social: o estado do amazonas em foco. VII Jornada Internacional de Políticas Públicas. Programa de Pós-graduação em políticas públicas. Universidade Federal do Maranhão, 2015.

SOBRAL, A.; FREITAS, C. M. Modelo de organização de indicadores para operacionalização dos determinantes socioambientais da saúde. Saúde e Sociedade, São Paulo, v. 19, n. 1, p. 35-47. 2010.

SUDAM. Diagnóstico e cenarização macrossocial da Amazônia legal: perfil da saúde na Amazônia legal e o contexto brasileiro. Belém, Pnud, 2000, 54 p.

VIANA, Rosana Lima; FREITAS, Carlos Machado de; GIATTI, Leandro Luiz. Saúde ambiental e desenvolvimento na Amazônia legal: indicadores socioeconômicos, ambientais e sanitários, desafios e perspectivas. Saúde e Sociedade, v. 25, p. 233246, 2016.

VICENTIN, G.; MINAYO, C. G. Gesundheit, Umwelt und wirtschaftliche Entwicklung im Amazonasgebiet. Science and Public Health, Rio de Janeiro, v. 8, n. 4 , p. 1069-1085, 2003.

WOLFE, N. Entwaldung, Jagd und die Ökologie der mikrobiellen Emergenz. Globaler Wandel \& Ähm. Gesundheit, 1(1):10-25, 2000.

Eingereicht: Dezember 2021.

Genehmigt: Dezember 2021 